\title{
Systematic Credit Risk: CDX Index Correlation and Extreme Dependence
}

\author{
Sofiane Aboura \\ Université de Paris-Dauphine, DRM-CEREG, France \\ Niklas Wagner \\ Passau University, Passau, Germany
}

September 12, 2007

\begin{abstract}
Dependence is an important issue in credit risk portfolio modeling and pricing. We discuss a straightforward common factor model of credit risk dependence, which is motivated by intensity models such as Duffie and Singleton (1998), among others. In the empirical analysis, we study dependence under the risk-neutral measure using credit default swap (CDS) spread data of liquid large-cap U.S. obligors. The proxy for the common factor is the DJ CDX.NA.IG index. We document that (i) the CDX factor is significant but has low explanatory power, (ii) factor sensitivities show distinct time-varying nature and that (iii) systematic credit risk shows asymmetric extreme factor dependence, where extreme dependence is pronounced for upward CDX movements. This finding from an EVT-copula approach is what is predicted by various intensity models of joint defaults.
\end{abstract}

Forthcoming in: Wagner N. (ed.) (2008): Credit Risk-Models, Derivatives and Management, Financial Mathematics Series Vol. 6, Chapman\&Hall/CRC, Boca Raton, London, New York. Acknowledgements: Part of the paper was written while the authors met at Leibniz University, Hannover. Estimations were partly obtained in R, A Language and Environment for Statistical Computing, Vienna, 2003. Corresponding author: Niklas Wagner, Passau University, Chair in Finance and Financial Control, 94032 Passau, Germany. E-mail: niklas.wagner@unipassau.de. 


\section{Introduction}

Dependence, including correlation, is an important issue in credit risk portfolio modelling and pricing. Credit portfolio derivatives such as collateralized debt obligations and index tranches can not be priced without a precise characterization of dependence, which allows for a derivation of portfolio loss distributions. Among the dependence approaches to credit risk that are documented in the literature, ${ }^{1}$ copula and factor approaches are two dominant concepts. First, the statistical copula approach as suggested for credit risk dependence modeling for example by Li (1999) allows for general representations of dependence, offering a highly flexible method, while the choice of a suitable copula function often seems crucial, see also e.g. Bluhm and Overbeck (2007). An empirical study on copula functions in modeling default dependence is by Das and Geng (2004). Second, the statistical factor approach as applied in credit risk modeling for example by Vasicek (1987), is a simplified dependence approach that allows for parsimonious and robust implementations, while it may suffer from over-simplification. While several authors argue that factor approaches may not be able to match observed dependence, Yu (2003) shows that factor approaches that take common risk factors of firm-specific credit risks into account may not be subject to this shortcoming.

In this contribution we address systematic credit risk, which is probably the most striking manifestation of credit risk dependence. Systematic credit risk was previously studied in a different setting e.g. by Pedrosa and Roll (1998). We discuss a straightforward common factor model of credit risk dependence. The model follows from the class of intensity models of credit risk, which model dependence in the driving intensity process. This is motivated in part by factor approaches as in Duffie and Singleton (1998), Yu (2003) and van der Voort (2004), for example.

A number of previous studies dealt with the phenomenon of dependent default events in credit portfolios. To this aim, structural credit risk models typically model dependence in latent underlying asset values (often proxied by equity values) of the obligors. While Lucas (1995) uses historical data whose availability is limited, Zhou (2001) analyses dependence in a bivariate structural Merton-

\footnotetext{
${ }^{1}$ For overviews on default dependence modeling see for example Duffie and Singleton (2003), Chapter 10, and Schlögl (2002). Using market variables, we may distinguish (i) rating and default correlations, (ii) spread correlations, (iii) asset or equity correlations and (iv) implied correlations. Since default events are rare events by definition, historical data are sparse. Therefore, latent credit risk dependence modeling approaches dominate.
} 
model. De Servigny and Renault (2002) study both equity-based and historical default correlations. They conclude that there does not seem to be an obvious link between the two. Das, Freed, Geng and Kapadia (2006) point out that in the Merton-model, correlation and time-variation in single firm equity values and equity volatilities will cause variation in default probabilities. Accordingly, they find that joint default probabilities are related to varying debt ratios, but are especially driven by varying correlated volatilities. Das, Duffie, Kapadia and Saita (2007) provide evidence that the majority of joint default risk is due to covariation in firm-specific default probabilities. As their results suggest, observable macroeconomic variables (including e.g. a business cycle variable) and particularly latent common variables play a major role in explaining correlated defaults.

We empirically study credit risk dependence under the risk-neutral measure, i.e. we consider derivatives prices and study their dependence implications. As pointed out in the preface to this volume, the market for credit default swap (CDS) contracts has grown enormously during the last couple of years. Hence, data for a study of credit risk dependence have recently become available through the derivatives markets. A study of credit risk dependence under the risk-neutral measure appears relevant as it reveals how credit risk is priced and traded in the market and it also forms the building block for the risk-neutral distribution of credit portfolios which serve as an underlying for other derivatives.

We study the dependence between index and component spread changes in our common factor model using liquid quotes from the U.S. credit default swap market during the years 2004 to 2006. In a first step we argue that CDS spread changes show distinct time-series dynamics, which should be taken into account in advance of studying dependence. As such we then consider factor dependence of unpredictable spread changes. We find that the CDX factor is significant for the chosen sample of large-cap U.S. obligors but has low explanatory power. Additionally, we shed light on a heavily time-varying nature of factor sensitivities. Various intensity models of joint defaults (see Section 2.2.4) predict positive jumps - i.e. large positive changes - in credit spreads conditional on the default of one or several obligors. In our setting, this translates into upper tail extreme factor dependence, a feature which is observable for most of our obligors.

The remainder of this chapter is organized as follows. Section 2 presents our methodology, which includes the derivation of the factor model, its implications and the methods used for inference. Section 3 outlines our empirical findings. The contribution ends with a brief conclusion in Section 4 . 


\section{Methodology}

Starting with the intensity based (or reduced form) approach to credit risk modeling, this section outlines a straightforward empirical model specification of common factor systematic credit risk under a risk-neutral measure. For a comprehensive summary of the model background and further literature see for example Duffie and Singleton (2003).

\subsection{Factor Intensity Approach}

In order to model default probabilities under risk-neutrality, where $\mathbb{Q}$ denotes the equivalent martingale measure, intensity models introduce a latent intensity process $\left(\lambda_{i, t}\right)_{0 \leq t \leq T}$ of the $i$ th obligor. Under standard assumptions, it follows that the conditional probability of default between time $t$ and $T$ is given by

$$
p_{i, t, T}^{\mathbb{Q}}=1-\mathbb{E}_{t} \exp \left(-\int_{t}^{T} \lambda_{i, s} d s\right) .
$$

A typical simplifying assumption is that the risk-neutral expected loss given default rate, "loss given default" in short, is constant and denoted by $l_{i}$. Then, the credit spread is simply given as:

$$
S_{i, t}=l_{i} \lambda_{i, t} .
$$

In order to introduce credit risk dependence of the obligors, we apply a common factor specification. A one-factor intensity model of credit risk dependence, in parts related e.g. to Duffie and Singleton (1998), Yu (2003) and van der Voort (2004), is given by

$$
\lambda_{i, t}=\lambda_{F, t}+\lambda_{i, t}^{\epsilon},
$$

where $\lambda_{F, t}$ and $\lambda_{i, t}^{\epsilon}$ are two independent intensity processes. Assigning any timevariation in the spread (2) to the time-varying intensity $\lambda_{i, t}$ only, ${ }^{2}$ a simple model of the credit spread dynamics follows. Fixing $S_{i, 0}$ and $\left(\lambda_{F, 0}, \lambda_{i, 0}\right)$, we can define the spread process $\left(S_{i, t}\right)_{0 \leq t \leq T}$ via the equation

$$
d S_{i, t}=l_{i} d \lambda_{F, t}+l_{i} d \lambda_{i, t}^{\epsilon} .
$$

\footnotetext{
${ }^{2}$ This assumption can be made without loss of generality since the intensity process is latent.
} 
Fixing also $S_{F, 0}$, we obtain for the factor spread, $d S_{F, t}=l_{F} d \lambda_{F, t}$, and therefore

$$
d S_{i, t}=l_{i} / l_{F} d S_{F, t}+l_{i} d \lambda_{i, t}^{\epsilon}
$$

We know from a broad body of empirical literature that observable spread changes are driven by various additional factors which are not related to default risk; see e.g. Collin-Dufresne, Goldstein and Martin (2001). However, equation (5) forms a fundamental model, which (i) shows how spread changes are driven by changes in intensity, (ii) models default dependence via a common factor intensity $\lambda_{F, t}$, and finally (iii) can easily be extended by taking other credit spread determinants into account.

\subsection{Factor Model Analysis}

In a discrete-time empirical setting, (5) translates into a regression model of the form

$$
\Delta S_{i, t}=a_{i}+b_{i} \Delta S_{F, t}+\epsilon_{i, t}, \quad t=1, \ldots, T,
$$

where the usual assumptions apply and the innovations are independent with identical distribution, $\epsilon_{i, t} \sim\left(0 ; \sigma_{i}^{2}\right)$.

In this setting, we see from (5) and (6) that systematic credit risk as measured via $b_{i}$ relates to individual and factor loss given default $l_{i}$ and $l_{F}$, with $b_{i}=l_{i} / l_{F}$. Hence, systematic credit risk is related to the $i$ th obligor's relative expected loss given default. There are various econometric approaches whose application can be motivated by the simple model in equation (6). Of course, a straightforward application is an ordinary least squares estimation of the model. In the following, we discuss how we use the model as a starting point for our analysis of crosssectional credit risk dependence.

Following (6), our focus is on the dependence between changes in the factor and the single obligor spreads. However, univariate analysis is a necessary and important step in going beyond a standard regression application and also necessary for a cross-sectional dependence study. Our following analysis considers four areas:

- univariate time series dependence in spread changes $\Delta S_{t}$,

- univariate extreme behavior of spread changes $\Delta S_{t}$,

- time-varying factor sensitivity of spread changes $\Delta S_{i, t}$, 
- extreme factor dependence of spread changes $\Delta S_{i, t}$.

\subsubsection{Time Series Dependence}

As univariate spread changes $\Delta S_{t}$ are driven by various factors including liquidity effects, ${ }^{3}$ it appears promising to account for liquidity effects at least within a linear time series model. Additionally, there is evidence that non-linear effects of volatility clustering are observable in spread changes as well; see e.g. Wagner, Hogan and Batten (2005). In order to allow for lagged responses to previous innovations, we may assume a model of the $\operatorname{ARMA}(1,1)$-form

$$
\Delta S_{t}=\mu+\varphi \Delta S_{t-1}+\phi U_{t-1}+U_{t}, \quad t=1, \ldots, T .
$$

Furthermore, the unpredictable spread changes, $U_{t}$, may have a time-varying conditional variance as given by a $\operatorname{GARCH}(1,1)$-specification

$$
\begin{aligned}
U_{t} & =\sigma_{t} Z_{t}, \\
\sigma_{t}^{2} & =\omega_{0}+\omega_{1} U_{t-1}^{2}+\omega_{2} \sigma_{t-1}^{2}, \quad \omega_{0}>0, \omega_{1}, \omega_{2} \geq 0,
\end{aligned}
$$

which is based on given start random variables $\left(\sigma_{0}^{2}, Z_{0}\right)$; see e.g. Bollerslev et al. (1992). The $Z_{t}$ 's are standardized iid random variables with common distribution function $F_{Z}$, where $Z_{t} \sim(0 ; 1)$. They represent standardized unpredictable spread changes.

\subsubsection{Extreme Behavior}

In order to characterize the extreme behavior of the univariate spread changes $\Delta S_{t}$, we may assume that the distribution function $F_{Z}$ of the innovations $Z_{t}$ in (8) is fat-tailed. Extreme value theory (EVT, see e.g. Embrechts, Klüppelberg and Mikosch (1997) or Coles (2001)) shows that this is equivalent to assuming that $F_{Z}$ has Pareto-like upper and lower tail

$$
\begin{aligned}
1-F_{Z}(z) & \sim c_{U} z^{-1 / \xi_{U}}, c_{U}>0, \xi_{U}>0, \\
F_{Z}(-z) & \sim c_{L} z^{-1 / \xi_{L}}, c_{L}>0, \xi_{L}>0, \quad \text { as } \quad z \rightarrow \infty .
\end{aligned}
$$

The parameters $\xi_{U}$ and $\xi_{L}$ denote the tail index of the upper tail and the lower tail, respectively.

\footnotetext{
${ }^{3}$ Note that liquidity effects typically relate to liquidity differentials between the treasury bond, the corporate bond and the credit derivatives market.
} 
We specify Pareto tail approximations for excesses of a sufficiently high positive threshold for example via the Generalized Pareto Distribution (GPD). Choosing two thresholds in our case, namely $v_{U}>0$ and $v_{L}>0$, we specify GPDdistributions for a random number of positive excesses of the upper threshold, $Y_{U}=Z-v_{U}>0$, and the lower threshold, $Y_{L}=-Z-v_{L}>0$, respectively:

$$
\begin{aligned}
1-F_{Z}\left(z-v_{U}\right) & =\left(1+\xi_{U} \frac{z-v_{U}}{\beta_{U}}\right)^{-1 / \xi_{U}}, \beta_{U}>0 \\
F_{Z}\left(-z-v_{L}\right) & =\left(1-\xi_{L} \frac{z-v_{L}}{\beta_{L}}\right)^{-1 / \xi_{L}}, \beta_{L}>0 .
\end{aligned}
$$

A characterization of the extreme behavior of spread changes allows for the modeling of so-called "gapping risk" (or jump risk) in credit spreads. This denotes the risk of large sudden spread changes, for example a sudden widening in spreads of more than 20 basis points for an index or more than 100 basis points for a single obligor; see e.g. Duffie and Singleton (2003, p. 138), and Bluhm and Overbeck (2007, p. 301). Previous studies that apply EVT in credit risk modeling include Phoa (1999), Campbell and Huisman (2002) and Lucas, Klaassen, Spreij and Straetmans (2001).

\subsubsection{Time-Varying Factor Sensitivity}

Our model approach in equation (6) assumes for simplicity that systematic credit risk, $b_{i}=l_{i} / l_{F}$, is constant. One extension to the model which may prove suitable in modeling credit risk, is to allow for a time-varying factor sensitivity, $b_{i, t}$ of the unpredictable spread changes $U_{t, i}$.

Allowing again for time-varying marginal spread change volatilities, $\sigma_{i, t}$ and $\sigma_{F, t}$, as in (8), multivariate autoregressive conditional heteroskedasticity offers a convenient model approach. We choose the diagonal BEKK-specification by Engle and Kroner (1995). In a bivariate setting, we can model individual and factor spread changes simultaneously, where a generalization of the standard relation for the correlation coefficient holds, yielding:

$$
b_{i, t}=\rho_{i, F, t} \frac{\sigma_{i, t}}{\sigma_{F, t}} .
$$

Hence, the time-varying conditional factor sensitivity $b_{i, t}$ is defined by the timevarying conditional correlation as well as the conditional spread change volatilities. 
Supporting the above specification, the empirical credit risk literature finds evidence of time-varying credit risk correlations. See for example the studies by Zhou (2001), de Servigny and Renault (2002) and Das, Freed, Geng and Kapadia (2006).

\subsubsection{Extreme Factor Dependence}

In order to characterize the joint extreme behavior of the spread changes $\Delta S_{t, i}$ and $\Delta S_{t, F}$, we may assume that the extreme behavior of the joint distribution function $F$ of the innovations $Z_{t, i}$ and $Z_{t, F}$ can be characterized via the Pickands EVT-copula approach; see e.g. Coles (2001). ${ }^{4}$

The Pickands representation theorem shows that a potential limiting distribution $H$, satisfying the required max-stability condition, has to be of the form

$$
H\left(z_{i}, z_{F}\right)=\exp \left\{-\left(\frac{1}{z_{i}}+\frac{1}{z_{F}}\right) A\left(\frac{z_{i}}{z_{i}+z_{F}}\right)\right\}, \quad z_{i}>v_{U, i}, z_{F}>v_{U, F} .
$$

Here, $H$ has a unique EVT-copula which is defined via the dependence function $A(w):[0,1] \mapsto[0,1]$, where $w=z_{i} /\left(z_{i}+z_{F}\right)$. $A(w)$ characterizes bivariate extreme dependence. If $A(w)=1$, the tails of the joint distribution are independent, whereas $A(w)=\max (w, 1-w)$ indicates perfect dependence. We choose three different parametric models for $A(w)$. A standard model is the symmetric logistic model, which relates to the Gumbel copula, where

$$
A_{\alpha}(w)=\left\{(1-w)^{1 / \alpha}+w^{1 / \alpha}\right\}^{\alpha},
$$

with $0<\alpha \leq 1$. Here, independence is reached when $\alpha=1$ and perfect dependence when $\alpha \rightarrow 0$. A generalization of the Gumbel copula allows for asymmetric dependence with

$$
A_{\alpha, \theta_{1}, \theta_{2}}(w)=\left(1-\theta_{1}\right)(1-w)+\left(1-\theta_{2}\right) w+\left\{(1-w)^{1 / \alpha} \theta_{1}^{1 / \alpha}+w^{1 / \alpha} \theta_{2}^{1 / \alpha}\right\}^{\alpha},
$$

where $0<\alpha \leq 1$ and $\theta_{1} \geq 0, \theta_{2} \leq 1$. Under this asymmetric model, symmetry is obtained under $\theta_{1}=\theta_{2}=1$ and independence is reached when either $\alpha=1$, $\theta_{1}=0$ or $\theta_{2}=0$. The asymmetric negative logistic model defines the GalambosJoe copula and is given by

$$
A_{\alpha, \theta_{1}, \theta_{2}}(w)=1-\left\{\left[(1-w) / \theta_{1}\right]^{-\alpha}+\left[w / \theta_{2}\right]^{-\alpha}\right\}^{-1 / \alpha},
$$

\footnotetext{
${ }^{4}$ For applications of the Pickands EVT-copula approach in finance see e.g. Longin and Solnik (2001) and Marsh and Wagner (2000).
} 
with $\alpha>0$ and $\theta_{1}>0, \theta_{2} \leq 1$. Independence is reached when either $\alpha \rightarrow 0$ or $\theta_{1} \rightarrow 0, \theta_{2} \rightarrow 0$.

For each of the above candidate dependence functions, it is possible to derive the limiting conditional exceedance probabilities

$$
\begin{aligned}
& \chi_{U}=\lim _{v_{U} \rightarrow \infty} P\left(Z_{i}>v_{U} \mid Z_{F}>v_{U}\right), \\
& \chi_{L}=\lim _{v_{L} \rightarrow \infty} P\left(-Z_{i}>v_{L} \mid-Z_{F}>v_{U}\right) .
\end{aligned}
$$

These limiting conditional probabilities, $\chi_{U}$ and $\chi_{L}$, indicate the presence of upper and lower factor tail dependence, respectively. For example, we may interpret $\chi_{U}$ as the probability of a significant jump in the spread of the $i$ th obligor given that the factor spread shows a significant jump, where both jump sizes exceed a very high threshold.

On the theory side various models, including e.g. Davis and Lo (2001), Jarrow and Yu (2001), Schönbucher and Schubert (2000) and Duffie, Eckner, Horel and Saita (2006), predict that the default of one obligor may cause other obligors' spreads to jump. Empirical evidence supporting this behavior is by CollinDufresne, Goldstein and Helwege (2003) and Zhang (2004), for example. The risk of individual spreads widening sharply in a state where spreads generally increase, results in the presence of spread jump dependence between our common factor and single spreads. For increasingly large jumps, this would predict upper factor tail dependence in our setting.

\section{Empirical Analysis}

\subsection{The CDS Data Set}

We study the common factor model of Section 2 based on a sample of credit default swap (CDS) data. The data comes from the Open Bloomberg system. The CDS obligors that we pick are eleven large-cap U.S. corporations taken from the 6th revision of the Dow Jones CDX Investment Grade (DJ CDX.NA.IG) index. The latter index universe consists of 125 U.S. corporations where CDSs are actively quoted. Hence, our sample assures a high level of market liquidity.

Our sample contains 625 CDS quotes for the index and for each corporation during the period January 9, 2004 to July 7, 2006. The quoted CDX index spread serves as a proxy for our common factor (CDX). The eleven individual 
corporations in our sample are: Altria Group Inc. (MO), American International Group Inc. (AIG), Boeing Co. (BA), Caterpillar Inc. (CAT), Dow Chemical Co. (DOW), Honeywell International Inc. (HON), Hewlett-Packard Co. (HPQ), International Business Machines Corp. (IBM), Mariott International Inc. (MAR), Motorola Inc. (MOT) and Walt Disney Co. (DIS). The CDS quotes are averages between quoted bid and ask prices as actual transaction prices are not publicly available. All quote series with the exception of BA and HPQ were obtained as complete series. In the case of BA and HPQ, there were two missing quotes, respectively. These missing data were linearly interpolated.

Spread changes are modeled as relative spread changes, $\Delta S_{t}=\ln C D S_{t}-$ $\ln C D S_{t-1}$, due to resulting unbounded support and other statistical properties (see also e.g. a note in Wagner, Hogan and Batten (2005)). A preliminary analysis of the spread change series gives summary statistics results as presented in Table 1. Mean values indicate that spreads were decreasing on average in the sample period. Spread change standard deviations are in a range between 0.021 for MAR and 0.034 for AIG, with 0.023 for the CDX index, indicating a remarkable annualized index volatility of 36.4 percent. Generally speaking, distributions tend to be positively skewed with excess kurtosis and the spread change observations show negative autocorrelation.

(Table 1 about here)

\subsection{Results}

The findings as summarized in Table 1 indicate that standard approaches to CDS spread risk and factor dependence modeling are prone to failure and more detailed time-series, tail and tail dependence analysis should apply. The presentation of our estimation results refers to the methods of Section 2.2.

In the first step we estimate the time-series model of Section 2.2.1 for our eleven obligors and the CDX via the standard maximum-likelihood (ML) method. It can be noted that all GARCH parameter estimates $\left(\omega_{1}, \omega_{2}\right)$ turn out to be significant at the 99 percent level for all series. At least one of the ARMA parameter estimates $(\varphi, \phi)$ is significant at the 90 percent level for all but CAT, MO and CDX index spread changes. ${ }^{5}$ This finding generally underlines the distinct time-series

\footnotetext{
${ }^{5}$ We therefore leave the results unreported and deliver them upon request.
} 
properties of CDS spread changes. We study unpredictable spread changes in the sequel.

In the second step we postpone extreme behavior and consider the factor model (6). We estimate least squares constant factor sensitivities and ML-BEKK timevarying factor sensitivities as in Section 2.2.3. A summary of our results is given in Table 2.

(Table 2 about here)

As we can see from Table 2, CDS spread changes are significantly related to CDX index spread changes with all coefficient estimates positive and significant at the 95 percent level. As our sample consists of high quality investment grade obligors, the coefficients' absolute values are relatively low and in a range between 0.082 (for IBM) and 0.205 (for DIS). Still, we have to attest that the results document weak overall explanatory power of our simple one-factor model: Rsquared statistics are in a range between 0.7 percent (for IBM) and 4.2 percent (for DIS). This finding illustrates that the model lacks additional covariates that explain default risk dependence, see also Das, Duffie, Kapadia and Saita (2007). To some extent the finding may also be explainable by a non-linear impact of the factor on firm-specific credit risk: while common factor changes do hardly affect single obligor spreads most of the time, they become a driving force in some states of the economy. Time-varying factor sensitivities and the analysis of extreme dependence can shed more light on such a scenario.

Table 2 also reports results on the ML-BEKK time-varying factor sensitivities. The results show that factor sensitivities remarkably fluctuate for most of our sample corporations, including DIS, MAR, DOW, BA and MOT. In contrast, MO and HON show stable sensitivities. Figure 2 plots time-varying factor sensitivities for two of our sample companies, AIG and DIS. As can be seen in the plot, factor sensitivities heavily fluctuate around their mean values. As shown by the results in Table 2, DIS has a higher average factor sensitivity than AIG. For short periods of time, DIS sensitivity regularly jumps to values as high as 0.35 to 0.70 while AIG sensitivity always remains below 0.21 .

(Figure 1 about here) 
In the third step we consider extreme spread change behavior. We use ARMAGARCH standardized residuals and then perform a simultaneous ML-estimation of the bivariate GPD model as described in Section 2.2.2 and 2.2.4. This method yields the marginal parameter estimates (see Section 2.2.2) as well as the dependence (or copula) parameter estimates (see Section 2.2.4), cf. Coles (2001). Based on mean residual life plots (or mean excess function plots, see e.g. Embrechts, Klüppelberg and Mikosch (1997)), we fix the marginal thresholds $v_{U}>0$ and $v_{L}>0$ for each series.

The GPD marginal parameter estimates yield estimated upper tail indices in the range of -0.081 (DOW) and 0.334 (MOT). Lower tail indices are between -0.233 (CAT) and 0.551 (DIS). These point estimate results do not obviously support fatter upper than lower tails. For the overall market, as represented by the CDX index we document moderate tail behavior (i.e. neither fat-tails nor thin-tails) for the lower as well as upper tail. This is also documented by the mean residual life plots in Figure 2 and Figure 3. Hence, our results support the hypothesis that the GARCH model captures non-normality in market spread changes. However, for some individual sample corporations, including AIG, DIS, HON, HPQ, IBM and MOT, we find upper tail index point estimates above 0.20, i.e. extreme risk for upward (adverse) spread residuals.

(Figure 2 about here)

(Figure 3 about here)

Our copula parameter estimation results for the upper tail are given in Table 3, those for the lower tail are given in Table 4 . We additionally report the conditional asymptotic probabilities $\chi_{U}$ and $\chi_{L}$ for the Gumbel copula model (14).

(Table 3 about here)

(Table 4 about here)

The results from Table 3 allow for the following conclusions. With the exception of HON and IBM, where the estimates of the dependence parameter $\alpha$ approach the limiting value of 1 , upper tail extreme dependence in CDS spread changes is present for the obligors. The corresponding conditional asymptotic 
probabilities $\chi_{U}$ can be interpreted as default probabilities given a major credit spread shock to the market: we see that AIG, DIS and MAR carry the largest extreme systematic credit risk with $\chi_{U}$ estimated to be larger than 10 percent. Evidence of an asymmetric dependence structure within the upper tail is weak. Table 3 shows that the asymmetric Gumbel copula (15) yields theta-parameter estimates close or equal to one in most cases and mostly estimates of similar magnitude. Only the cases of HPQ and MO may indicate some asymmetry. The Galambos-Joe copula (16) estimation results principally confirm our findings from the Gumbel copula estimations while the results show remarkably less stability.

The results from Table 4 show that the dependence parameter $\alpha$ closely approaches the value of 1 for all of the obligors. Hence, lower tail dependence is negligible and estimated factor dependence in large spread decreases for DIS and HON is minor. We may generally assume that spread changes are asymptotically independent (as e.g. under the bivariate normal model) and that the corresponding conditional asymptotic probabilities $\chi_{L}$ are zero. Again, evidence of an asymmetric dependence structure within the individual tails is not given.

Comparing the Gumbel copula estimation results in Table 3 (upper tail) and Table 4 (lower tail) suggests that extreme dependence is asymmetric when considering the upper versus the lower tail behavior. Extreme dependence appears as an issue with upward shocks to CDX spreads.

\section{Conclusion}

Systematic credit risk is driven by credit risk dependence. In this contribution we show that a common factor model of credit risk dependence has several interesting features. While common factor changes may hardly affect single obligors' spreads most of the time, they may become a driving force in some states of the economy. Time-varying factor sensitivities and the analysis of extreme factor dependence shows that obligors will have varying exposures to the market not only crosssectionally (as e.g. given by their rating class) but also during time. We emphasize that a time-varying nature of systematic credit risk goes along with asymmetric extreme dependence. These findings are important for portfolio diversification and for future research into asset pricing models of credit risk premia. 


\section{References}

[1] Bluhm C., Overbeck L. (2007): Structured Credit Portfolio Analysis, Baskets and CDOs, Chapman \& Hall CRC, Boca Raton, London, New York

[2] Bollerslev T., Chou R. Y., Kroner K. F. (1992): ARCH Modeling in Finance: A Review of the Theory and Empirical Evidence, Journal of Econometrics 52: 5-59

[3] Campbell R., Huisman R. (2002): Measuring Credit Spread Risk: Incorporating the Tails, Working Paper, Erasmus University Rotterdam

[4] Coles S. (2001): An Introduction to Statistical Modeling of Extreme Values, Springer, London

[5] Collin-Dufresne P., Goldstein R. S., Helwege J. (2003): Is Credit Event Risk Priced? Modeling Contagion via the Updating of Beliefs, Working Paper, U.C. Berkeley

[6] Collin-Dufresne P., Goldstein R. S., Martin J. S. (2001): The Determinants of Credit Spread Changes, Journal of Finance 56: 2177-2207

[7] Das S. R., Duffie D., Kapadia N., Saita L. (2007): Common Failings: How Corporate Defaults are Correlated, Journal of Finance 62: 93-117

[8] Das S. R., Freed L., Geng G., Kapadia N. (2006): Correlated Default Risk, Journal of Fixed Income 16: 7-32

[9] Das S. R., Geng G. (2004): Correlated Default Processes: A Criterion Based Copula Approach, Journal of Investment Management 2: 44-70 (reprinted as Chapter 19 of this volume)

[10] Davis M., Lo V. (2001): Infectious Default, Quantitative Finance 1: 382387

[11] de Servigny A., Renault O. (2002): Default Correlation: Empirical Evidence, Working Paper, Standard \& Poor's

[12] Duffie D., Eckner A., Horel G., Saita L. (2006): Frailty Correlated Default, Working Paper, Stanford University 
[13] Duffie D., Singleton, K. J. (1998): Simulating Correlated Defaults, Working Paper, Stanford University

[14] Duffie D., Singleton, K. J. (2003): Credit Risk: Pricing, Measurement, and Management, Princeton University Press, Princeton

[15] Embrechts P., Klüppelberg C., Mikosch T. (1997): Modelling Extremal Events for Insurance and Finance, Springer, New York

[16] Engle R. F., Kroner K. F. (1995): Multivariate Simultaneous Generalized ARCH, Econometric Theory 11: 122-150

[17] Jarrow R. A., Yu F. (2001): Counterparty Risk and the Pricing of Defaultable Securities, Journal of Finance 56: 1765-1799

[18] Li D. X. (1999): On Default Correlation: A Copula Function Approach, Working Paper, RiskMetrics, New York

[19] Lucas A., Klaassen P., Spreij P., Straetmans S. (2001): An Analytic Approach to Credit Risk of Large Corporate Bond and Loan Portfolios, Journal of Banking and Finance 25: 1635-1664

[20] Lucas D. J. (1995): Default Correlation and Credit Analysis, Journal of Fixed Income 5: 76-87

[21] Longin, F., Solnik, B. (2001): Extreme Correlation of International Equity Markets, Journal of Finance 56: 649-676

[22] Marsh, T. A., Wagner, N. (2000): Return-Volume Dependence and Extremes in International Equity Markets, Working Paper No. RPF-293, U.C. Berkeley

[23] Pedrosa, M., Roll, R. (1998): Systematic Risk in Corporate Bond Credit Spreads, Journal of Fixed Income, December 1998: 7-26

[24] Phoa W. (1999): Estimating Credit Spread Risk using Extreme Value Theory, Journal of Portfolio Management, Spring 1999: 69-73

[25] Schlögl E. (2002): Default Correlation Modelling, Technical Note, University of Technology, Sydney 
[26] Schönbucher P., Schubert D. (2000): Copula-Dependent Default Risk in Intensity Models, Working Paper, Bonn University

[27] van der Voort M. (2004): Double Default Correlation, Working Paper, Erasmus University Rotterdam

[28] Vasicek O. A. (1987): Probability of Loss on Loan Portfolio, Working Paper, KMV Corporation

[29] Wagner N., Hogan W., Batten J. A. (2005): Interest Rates, Stock Returns and Credit Spreads: Evidence from German Eurobonds, Economic Notes 34: 35-50

[30] Yu F. (2003): Default Correlation in Reduced-Form Models, Working Paper, U.C. Irvine

[31] Zhang G. (2004): Intra-Industry Credit Contagion: Evidence from the Credit Default Swap Market and the Stock Market, Working Paper, U.C. Irvine

[32] Zhou C. (2001): An Analysis of Default Correlation and Multiple Defaults, Review of Financial Studies 14: 555-576 
Table 1

Summary statistics for daily logarithmic CDS spread changes $\Delta S_{t}$.

\begin{tabular}{lcccccc} 
name & mean & std & skew & kurt & corr $_{t ; t-1}$ & corr $_{t ; t}-2$ \\
\hline \hline CDX & 0.000 & 0.023 & 0.402 & 10.124 & -0.018 & -0.011 \\
\hline AIG & 0.000 & 0.034 & 3.360 & 42.899 & 0.217 & 0.014 \\
BA & -0.002 & 0.030 & -0.304 & 5.817 & -0.190 & 0.111 \\
CAT & 0.000 & 0.028 & 0.381 & 6.867 & -0.194 & -0.024 \\
DIS & -0.001 & 0.032 & 0.044 & 16.606 & -0.111 & 0.160 \\
DOW & -0.001 & 0.030 & 0.455 & 8.995 & 0.022 & 0.057 \\
HON & -0.001 & 0.028 & 0.992 & 11.002 & -0.150 & -0.029 \\
HPQ & 0.000 & 0.032 & 0.158 & 12.965 & -0.196 & 0.119 \\
IBM & -0.001 & 0.027 & 2.277 & 26.671 & -0.162 & 0.072 \\
MAR & 0.000 & 0.021 & 0.684 & 10.031 & -0.084 & 0.078 \\
MO & -0.002 & 0.027 & 1.206 & 21.594 & 0.021 & 0.076 \\
MOT & -0.001 & 0.025 & -0.429 & 11.846 & 0.134 & 0.123 \\
\hline \hline
\end{tabular}


Table 2

Point estimates of factor sensitivities $b_{i}$ together with t-values and coefficients of determination, statistics on the BEKK time-varying factor sensitivities $b_{i, t}$.

\begin{tabular}{|c|c|c|c|c|c|c|}
\hline name & $b_{i}$ & t-value & $\mathrm{R}^{2}$ & $\min \left(b_{i, t}\right)$ & $\max \left(b_{i, t}\right)$ & $\operatorname{std}\left(b_{i, t}\right)$ \\
\hline AIG & 0.121 & 3.05 & 0.015 & -0.066 & 0.204 & 0.019 \\
\hline BA & 0.113 & 2.84 & 0.013 & -0.102 & 0.188 & 0.025 \\
\hline CAT & 0.146 & 3.67 & 0.021 & 0.112 & 0.262 & 0.010 \\
\hline DIS & 0.205 & 5.21 & 0.042 & -0.134 & 0.707 & 0.068 \\
\hline DOW & 0.159 & 4.03 & 0.025 & -0.109 & 0.252 & 0.033 \\
\hline $\mathrm{HON}$ & 0.088 & 2.19 & 0.008 & 0.061 & 0.084 & 0.003 \\
\hline HPQ & 0.135 & 3.41 & 0.018 & -0.098 & 0.138 & 0.013 \\
\hline IBM & 0.082 & 2.04 & 0.007 & -0.108 & 0.079 & 0.013 \\
\hline MAR & 0.133 & 3.33 & 0.018 & -0.152 & 0.251 & 0.037 \\
\hline MO & 0.115 & 2.89 & 0.013 & 0.109 & 0.117 & 0.001 \\
\hline MOT & 0.144 & 3.61 & 0.020 & -0.048 & 0.323 & 0.024 \\
\hline
\end{tabular}


Table 3

Upper tail ML-estimation results for the bivariate GPD EVT-copula model. * denotes parameter different from zero at the 95 percent level.

\begin{tabular}{lccccccccc}
\hline & $v$ & Gumbel & \multicolumn{3}{c}{ asy.-Gu. } & \multicolumn{5}{c}{ Galam. } \\
\hline \hline name & & $\alpha$ & $\chi \%$ & $\alpha$ & $\theta_{1}$ & $\theta_{2}$ & $\alpha$ & $\theta_{1}$ & $\theta_{2}$ \\
\hline \hline CDX & 1.00 & & & & & & & & \\
\hline AIG & 0.95 & $0.906^{*}$ & 12.6 & $0.911^{*}$ & $0.999^{*}$ & 0.748 & $0.489^{*}$ & $0.999^{*}$ & 0.313 \\
BA & 1.05 & $0.979^{*}$ & 2.9 & $0.999^{*}$ & 0.745 & 0.787 & $15.00^{*}$ & 0.0316 & 0.260 \\
CAT & 1.25 & $0.939^{*}$ & 8.3 & $0.956^{*}$ & 0.734 & $1.000^{*}$ & 7.25 & 0.0679 & 0.355 \\
DIS & 1.25 & $0.910^{*}$ & 12.1 & $0.908^{*}$ & $1.000^{*}$ & $0.741^{*}$ & $15.00^{*}$ & $0.192^{*}$ & 0.0730 \\
DOW & 1.00 & $0.939^{*}$ & 8.3 & $0.961^{*}$ & $0.999^{*}$ & 0.762 & 13.50 & $0.489^{*}$ & 0.0335 \\
HON & 1.25 & $0.999^{*}$ & 0.1 & $0.999^{*}$ & 0.775 & 0.987 & $0.200^{*}$ & 0.454 & 0.487 \\
HPQ & 1.50 & $0.945^{*}$ & 7.5 & 0.550 & 0.149 & 0.340 & 1.174 & 0.144 & 0.332 \\
IBM & 1.50 & $1.000^{*}$ & 0.1 & $0.999^{*}$ & 0.763 & 0.984 & $0.201^{*}$ & 0.454 & 0.631 \\
MAR & 1.50 & $0.911^{*}$ & 12.0 & 0.457 & $0.264^{*}$ & 0.161 & 1.496 & $0.254^{*}$ & 0.168 \\
MO & 0.85 & $0.961^{*}$ & 5.3 & $0.986^{*}$ & 0.734 & $1.000^{*}$ & 1.572 & 0.0607 & $0.679^{*}$ \\
MOT & 1.25 & $0.929^{*}$ & 9.6 & $0.947^{*}$ & 0.734 & $1.000^{*}$ & 0.790 & 0.177 & 0.414 \\
\hline \hline
\end{tabular}




\section{Table 4}

Lower tail ML-estimation results for the bivariate GPD EVT-copula model. * denotes parameter different from zero at the 95 percent level.

\begin{tabular}{|c|c|c|c|c|c|c|c|c|c|}
\hline & $v$ & Gumbel & & asy.-Gu. & & & Galam & & \\
\hline name & & $\alpha$ & $x^{0} \%$ & $\alpha$ & $\theta_{1}$ & $\theta_{2}$ & $\alpha$ & $\theta_{1}$ & $\theta_{2}$ \\
\hline CDX & 1.50 & & & & & & & & \\
\hline AIG & 1.50 & $1.000 *$ & 0.0 & $1.000^{*}$ & 0.789 & 0.782 & $0.201^{*}$ & 0.561 & 0.553 \\
\hline BA & 1.45 & $1.000 *$ & 0.0 & $0.999^{*}$ & 0.944 & 0.797 & 0.349 & 0.0169 & $1.000^{*}$ \\
\hline CAT & 1.50 & $1.000^{*}$ & 0.0 & $0.999^{*}$ & 0.811 & 0.801 & $0.200^{*}$ & 0.595 & 0.606 \\
\hline DIS & 1.95 & $0.964^{*}$ & 5.0 & $0.687^{*}$ & 0.053 & $0.999^{*}$ & 0.366 & 0.0985 & $1.000 *$ \\
\hline DOW & 0.25 & $0.988^{*}$ & 1.7 & $0.962^{*}$ & 0.001 & 0.941 & $0.200^{*}$ & 0.0350 & 0.552 \\
\hline $\mathrm{HON}$ & 1.50 & $0.969^{*}$ & 4.3 & $0.961^{*}$ & 0.712 & $1.000^{*}$ & 9.865 & 0.0572 & 0.119 \\
\hline HPQ & 1.55 & $0.999 *$ & 0.1 & $0.999^{*}$ & 0.706 & 0.955 & $0.200^{*}$ & 0.610 & 0.705 \\
\hline IBM & 1.00 & $0.999 *$ & 0.1 & $1.000^{*}$ & 0.826 & 0.883 & $0.200^{*}$ & 0.375 & 0.529 \\
\hline MAR & 0.75 & $0.985^{*}$ & 2.1 & $0.989^{*}$ & $0.999^{*}$ & 0.728 & 0.285 & $0.999^{*}$ & 0.0937 \\
\hline MO & 1.50 & $0.999^{*}$ & 0.1 & $1.000 *$ & 0.858 & 0.848 & $0.200 *$ & 0.569 & 0.565 \\
\hline MOT & 1.50 & $0.999^{*}$ & 0.1 & $1.000^{*}$ & 0.887 & 0.801 & $0.201^{*}$ & 0.646 & 0.627 \\
\hline
\end{tabular}




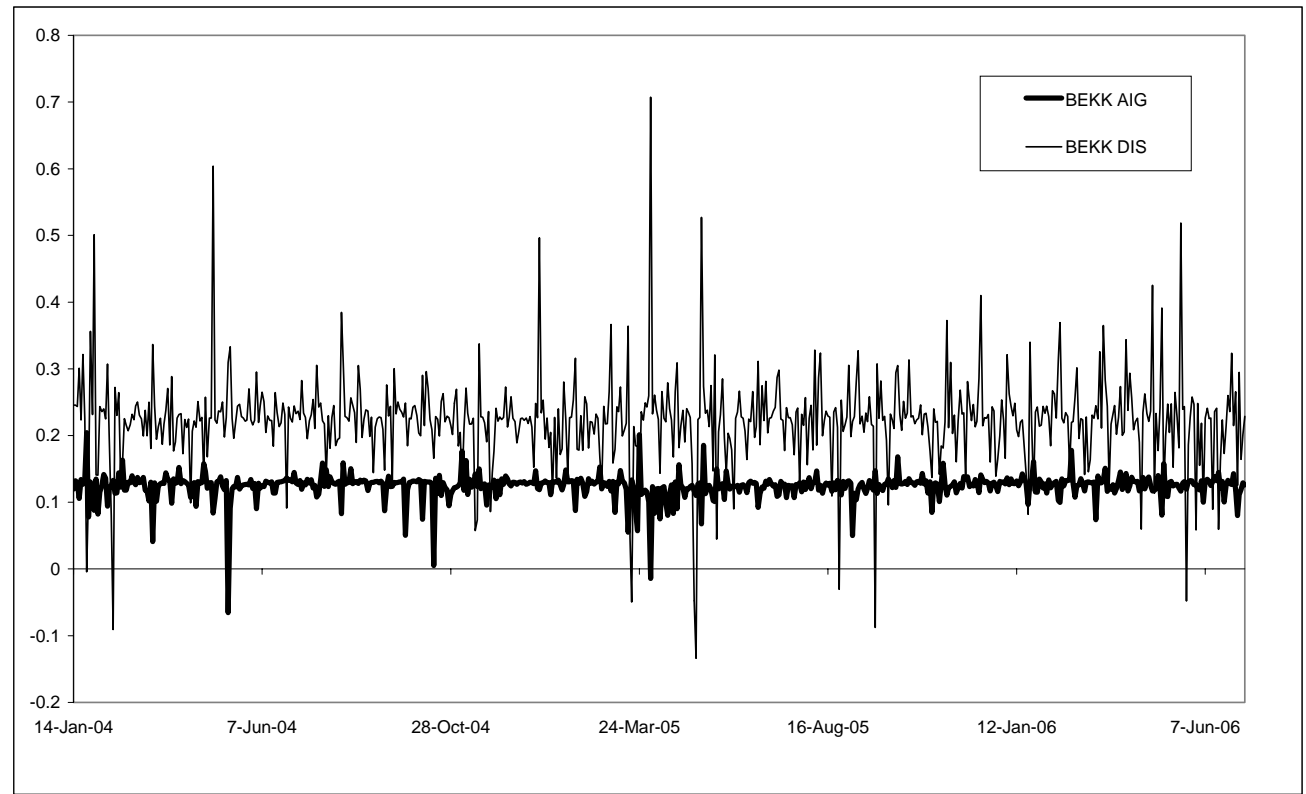

Figure 1: BEKK conditional CDX factor correlation for American International Group Inc. (AIG) and Walt Disney Co. (DIS). 


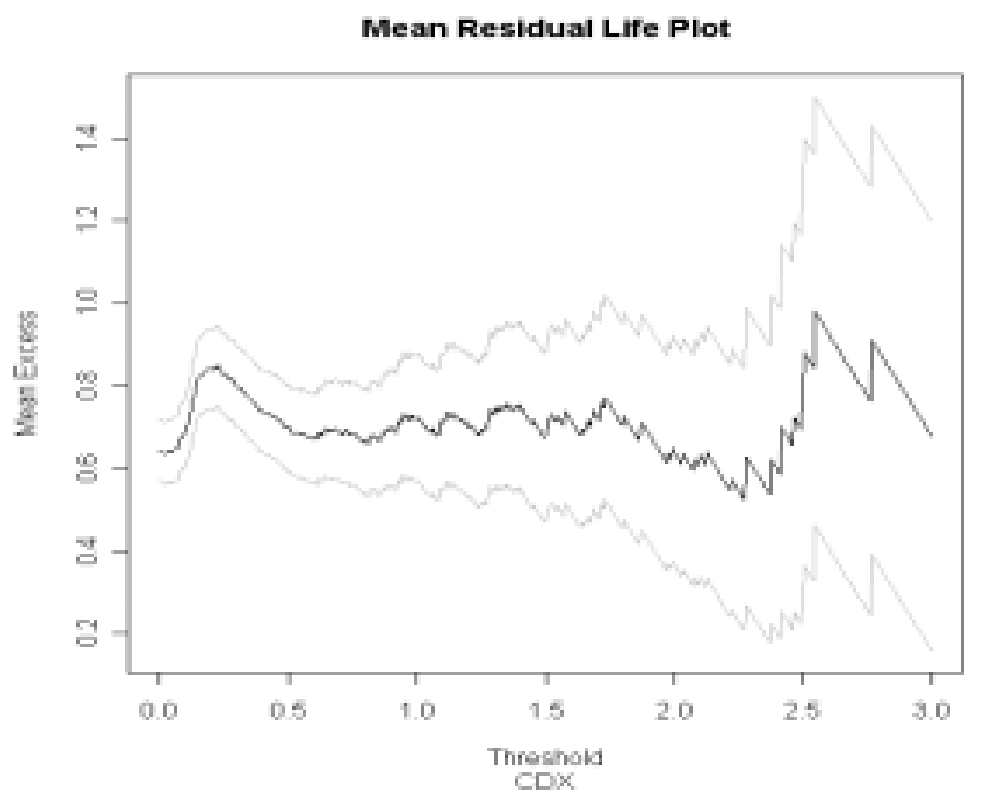

Figure 2: Mean excess function for upper tail CDX standardized unpredictable spread changes. 


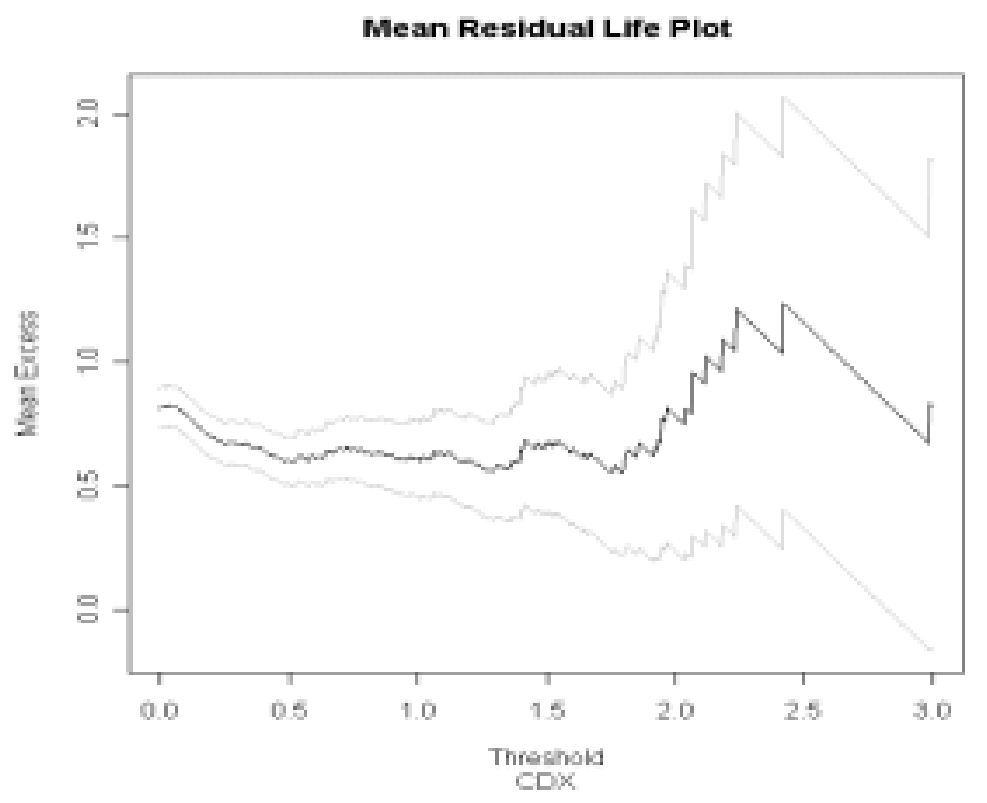

Figure 3: Mean excess function for lower tail CDX standardized unpredictable spread changes. 